Supporting Information for

\title{
4-Substituted Trinems as Broad Spectrum $\beta$-Lactamase Inhibitors: Structure-based Design, Synthesis and Biological Activity
}

Ivan Plantan, ${ }^{\dagger}$ Lovro Selič, ${ }^{\dagger}$ Tomaž Mesar, ${ }^{\dagger}$ Petra Štefanič Anderluh, ${ }^{\dagger}$ Marko Oblak, ${ }^{\dagger}$ Andrej Preželj, ${ }^{\dagger}$ Lars Hesse, ${ }^{\ddagger}$ Miha Andrejašič, ${ }^{\S}$ Mateja Vilar, ${ }^{\dagger}$ Dušan Turk, ${ }^{\S}$ Andrej Kocijan, ${ }^{\dagger}$ Tadeja Prevec, ${ }^{\dagger}$ Gregor Vilfan, ${ }^{\dagger}$ Darko Kocjan, ${ }^{\dagger}$ Anton Čopar, ${ }^{\dagger}$ Uroš Urleb, ${ }^{\dagger}$ and Tom Solmajer ${ }^{\dagger, \mathbb{I}, *, \dagger}$.

${ }^{\dagger}$ Lek Pharmaceuticals d.d., Drug Discovery, Verovskova 57, SI-1526 Ljubljana, Slovenia

II National Institute of Chemistry, POB 660, Hajdrihova 19, 1001 Ljubljana, Slovenia

* Sandoz, Antibiotic Research Institute, A-1235 Wien, Austria

$\S$ Jožef Stefan Institute, Department of Biochemistry and Molecular Biology, Jamova 39, SI-1000 Ljubljana, Slovenia

e-mail: tom.solmajer@ki.si

\section{Table of Contents}

\begin{tabular}{|l|l|l|l}
\hline Table S1: Analytical data for determining the purity of target compounds & S-2
\end{tabular}

Table S2. Crystallographic data collection and structure refinement statistics.

S-3

General Procedure A. Synthesis of 3-(S)-[1-(R)-(tert-Butyldimethylsilyloxy)-ethyl]-4$(R)-((1 S / R)-(3 R / S)$-alkoxy-2-oxo-cyclohexyl)-azetidin-2-one (4a-4f)

2. General Procedure B. Synthesis of 3-(S)-[1-(R)-(tert-Butyldimethylsilyloxy)-ethyl]-4$(R)-((1 R / S)-3-(S / R)$-alkoxy-2-oxo-cyclohexyl)-allyloxalyl-azetidin-2-on (7a-h)

3. General Procedure C. Synthesis of allyl $(8 S / 8 R, 9 R)-10-(S)-[1-(R)-($ tertbutyldimethylsilyloxy)-ethyl]-4-(S/R)-alkoxy-11-oxo-1-azatricyclo[7.2.0.0 $\left.0^{3,8}\right]$ undec-2-encarboxylate (8a-8h)

4. General Procedure D. Synthesis of allyl $(8 S / 8 R, 9 R)-10-(S)-[1-(R)$-hydroxyethyl]-4$(S / R)$-alkoxy-11-oxo-1-azatricyclo[7.2.0.0 $\left.0^{3,8}\right]$ undec-2-en-carboxylate (9a-9h)

5. General Procedure E. Synthesis of allyl $(8 S / 8 R, 9 R)-10-(E)$-ethylidene-4- $(S / R)$-alkoxy-11- S-8 oxo-1-azatricyclo[7.2.0.0 $\left.0^{3,8}\right]$ undec-2-en-carboxylate (10a-10h) 
Table S1: Analytical data for determining the purity of target compounds

\begin{tabular}{|c|l|l|c|c|}
\hline Compound & & $\mathbf{R}$ & HPLC (area \%) $^{\mathbf{a}}$ & HPLC (area \%) $^{\mathbf{b}}$ \\
\hline 11a & $(4 S, 8 S)$ & $\mathrm{R}=\mathrm{Me}$ & 99.55 & 100.0 \\
\hline $\mathbf{1 1 b}$ & $(4 R, 8 R)$ & $\mathrm{R}=\mathrm{Et}$ & 98.02 & 99.90 \\
\hline $\mathbf{1 1 c}$ & $(4 S, 8 S)$ & $\mathrm{R}=\mathrm{Et}$ & 98.00 & 99.90 \\
\hline $\mathbf{1 1 d}$ & $(4 S, 8 S)$ & $\mathrm{R}=\mathrm{iPr}$ & 98.86 & 98.45 \\
\hline $\mathbf{1 1 e}$ & $(4 R, 8 R)$ & $\mathrm{R}=\mathrm{Bu}$ & 98.69 & 98.76 \\
\hline $\mathbf{1 1 f}$ & $(4 S, 8 S)$ & $\mathrm{R}=\mathrm{Bu}$ & 97.03 & 96.81 \\
\hline $\mathbf{1 1 g}$ & $(4 S, 8 S)$ & $\mathrm{R}=\left(\mathrm{CH}_{2}\right)_{2} \mathrm{~F}$ & 98.20 & 99.90 \\
\hline $\mathbf{1 1 h}$ & $(4 S, 8 S)$ & $\mathrm{R}=\left(\mathrm{CH}_{2}\right)_{2} \mathrm{CN}$ & 97.00 & 97.92 \\
& & & & \\
\hline
\end{tabular}

a- HPLC analyses were performed using a Waters 2695 Separation Module with a Waters 2996 PDA detector and an XTerra RP C18 (150x4.6 mm I.D., $3.5 \mu \mathrm{m}$ particle size) analytical column and a gradient elution method combining mobile phase A with $25 \mathrm{mM}$ ammonium acetate $(\mathrm{pH}=6) /$ acetonitrile $(95 / 5$ $\mathrm{v} / \mathrm{v})$ and mobile phase $\mathrm{B}$ with $25 \mathrm{mM}$ ammonium acetate $(\mathrm{pH}=6) /$ acetonitrile $(10 / 90 \mathrm{v} / \mathrm{v})$.

b- alternative additional HPLC analyses were performed (Method 1 in Experimental Section) using Agilent 1100 Separation Module and Luna C18 (250x4.6 mm I.D., 5mm particle size) analytical column by an isocratic elution method combining $25 \mathrm{mM}$ ammonium phosphate buffer $(\mathrm{pH}=7.57) /$ acetonitrile $(60 / 40$ $\mathrm{v} / \mathrm{v})$. 
Table S2. Crystallographic data collection and structure refinement statistics.

\begin{tabular}{|l|l|l|}
\hline Enzyme complex & $\mathbf{1 1 a}$ & $\mathbf{1 1 e}$ \\
\hline Space group & $\mathrm{P} 2{ }_{1}{ }_{1} 2$ & $\mathrm{P}{ }_{1}{ }_{1} 2$ \\
\hline Cell dimensions $(\AA)$ & $76.652,69.776,62.818$ & $77.327,68.984,62.020$ \\
& $90,90,90$ & $90,90,90$ \\
\hline Temp $(\mathrm{K}), \lambda(\AA)$ & $100,1.00$ & $100,1.5418$ \\
\hline $\mathrm{d}_{\text {min }}($ highest shell $)(\AA)$ & $30.00-2.05(2.09-2.05)$ & $50.00-2.20(2.28-2.20)$ \\
\hline Observations & 190923 & 60033 \\
\hline Unique reflections & 21206 & 17447 \\
\hline Completeness $(\%)$ & $96.8(84.9)$ & $90.7(64.7)$ \\
\hline Avg I/ $(\mathrm{I})$ & $9.15(2.08)$ & $14.21(2.47)$ \\
\hline $\mathrm{R}_{\text {sym }}(\mathrm{I})$ & $0.121(0.321)$ & $0.068(0.284)$ \\
\hline R factor & 0.209 & 0.263 \\
\hline R-free & 0.245 & 0.315 \\
\hline Bond RMS & 0.012 & 0.009 \\
\hline Angle RMS & 1.686 & 1.744 \\
\hline Number of atoms & 4450 & 3145 \\
\hline Number of solvent molecules & 341 & \\
\hline B-factor & 22.07 & \\
\hline & & \\
\hline
\end{tabular}




\section{General Procedure A. Synthesis of 3-(S)-[1-(R)-(tert-Butyldimethylsilyloxy)-ethyl]-4-(R)-((1S/R)-(3R/S)-}

alkoxy-2-oxo-cyclohexyl)-azetidin-2-one (4a-4f)

A solution of 2-alkoxycyclohexanone (2a-d) (13.2 mL, 1.2 EQ) in dry dichloromethane (150 mL) was cooled to $-20^{\circ} \mathrm{C}$. $\mathrm{SnCl}_{4}(35 \mathrm{~mL}, 3.4 \mathrm{EQ})$ was added dropwise at $-20^{\circ} \mathrm{C}$ and the reaction mixture was stirred for an additional $15 \mathrm{~min}$ at $-20^{\circ} \mathrm{C}$. Next, a solution of AZT (3) $((3 R, 4 R)-3-((1 R)-1-\{[$ tertbutyl(dimethyl)silyl]oxy\}ethyl)-4-acetoxy-2-azetidinone, $25 \mathrm{~g}, 1 \mathrm{EQ})$ in dry dichloromethane (75 mL) was added during vigorous stirring and the temperature was allowed to rise to $0^{\circ} \mathrm{C}$. The reaction mixture was stirred for an additional $1 \mathrm{~h}$ at $0^{\circ} \mathrm{C}$ and a solution of ethyl diisopropylamine (EDIPA, 40 $\mathrm{mL})$ in dichloromethame $(60 \mathrm{~mL})$ was subsequently added stepwise at $0^{\circ} \mathrm{C}$. After $1 \mathrm{~h}$ stirring at $0^{\circ} \mathrm{C}$ the reaction mixture was poured into Rochell solution $\left(\mathrm{NaCl}(25 \mathrm{~g}), \mathrm{NaHCO}_{3}(25 \mathrm{~g}), \mathrm{KNa}\right.$ tartrate $(25 \mathrm{~g})$ in $750 \mathrm{~mL}$ water) and ethyl acetate $(750 \mathrm{~mL})$ was added. The obtained mixture was stirred for $1 \mathrm{~h}$ at room temperature. The layers were separated and the organic phase was washed with Rochell solution and a saturated solution of $\mathrm{NaCl}$, dried with $\mathrm{MgSO}_{4}$ and the solvent evaporated under reduced pressure. The remaining oily residue was suspended in hexane $(100 \mathrm{~mL})$ and cooled to form a white precipitate, which was filtered off or purified using column chromatography. The single diastereoisomers were obtained by column chromatography or by crystallization of the obtained mixture.

3-(S)-[1-(R)-(tert-Butyldimethylsilyloxy)-ethyl]-4-(R)-((1S,3R)-3-butoxy-2-oxo-cyclohexyl)-azetidin-2- one $(4 e)$

Compound 4e was prepared according to General Procedure A, followed by separation of diastereomers by column chromatography (silica gel; ethyl acetate/hexane $=1 / 6 \rightarrow 1 / 2$ ); yield: $34.4 \%$, white crystals; m.p. 91.8$93.6^{\circ} \mathrm{C} ;[\alpha]_{\mathrm{D}}{ }^{20^{\circ} \mathrm{C}}\left(\mathrm{CH}_{2} \mathrm{Cl}_{2}, \mathrm{c}=1.02 \mathrm{mg} / \mathrm{ml}\right)=+15.1^{\circ} . \mathrm{IR}(\mathrm{KBr}) \vee\left[\mathrm{cm}^{-1}\right] 777,837,1118,1708,1759,2931,3348$. ${ }^{1} \mathrm{H}$ NMR $\left(\mathrm{CDCl}_{3}\right) \delta[\mathrm{ppm}] 0.03\left(\mathrm{~s}, 3 \mathrm{H}, \mathrm{CH}_{3} \mathrm{Si}\right), 0.04\left(\mathrm{~s}, 3 \mathrm{H}, \mathrm{CH}_{3} \mathrm{Si}\right), 0.84(\mathrm{~s}, 9 \mathrm{H}, t-\mathrm{BuSi}), 0.88(\mathrm{t}, 3 \mathrm{H}, J=7.3 \mathrm{~Hz}$, $\left.\underline{\mathrm{CH}}_{3}-\left(\mathrm{CH}_{2}\right)_{3}-\mathrm{O}\right), 1.21\left(\mathrm{~d}, 3 \mathrm{H}, J=6.3 \mathrm{~Hz}, \underline{\mathrm{CH}}_{3}-\mathrm{CH}-\mathrm{OSi}\right), 1.26-1.70(\mathrm{~m}, 7 \mathrm{H}), 1.90-2.26(\mathrm{~m}, 3 \mathrm{H}), 2.82(\mathrm{dd}, 1 \mathrm{H}$, $\left.J_{1}=2.3, J_{2}=5.5 \mathrm{~Hz}, \mathrm{H}-3\right), 3.05\left(\mathrm{ddd}, 1 \mathrm{H}, J_{1}=4.5, J_{2}=5.7, J_{3}=12.2 \mathrm{~Hz}, \mathrm{H}-{ }^{‘} 1\right), 3.18-3.42\left(\mathrm{~m}, 2 \mathrm{H}, \mathrm{O}-\underline{\mathrm{CH}}_{2}-\left(\mathrm{CH}_{2}\right)_{2}-\mathrm{CH}_{3}\right)$, 
$3.61\left(\operatorname{degd}, 1 \mathrm{H}, J=3.2 \mathrm{~Hz}, \mathrm{H}-{ }^{\prime} 3\right), 3.93\left(\mathrm{dd}, 1 \mathrm{H}, J_{1}=2.3, J_{2}=4.1 \mathrm{~Hz}, \mathrm{H}-4\right), 4.0-4.25$ (deg. dq, $1 \mathrm{H}, \mathrm{J}=6 \mathrm{~Hz}, \mathrm{CH}_{3}-\underline{\mathrm{CH}}(-$ OTBS)-CH-), 6.13 (s, 1H, NH). HRMS ([M+H] $\left.]^{+}\right)$: Anal. calcd. for $\mathrm{C}_{21} \mathrm{H}_{40} \mathrm{NO}_{4} \mathrm{Si}$ : 398.2727; found: 398.2721 . 3-(S)-[1-(R)-(tert-Butyldimethylsilyloxy)-ethyl]-4-(R)-((1R,3S)-3-butoxy-2-oxo-cyclohexyl)-azetidin-2-one (4f)

Compound 4f was prepared according to General Procedure A followed by separation of the diastereomers by column chromatography (silica gel; ethyl acetate/hexane $=1 / 6 \rightarrow 1 / 2$ ); yield: $42.2 \%$, white crystals; m.p. 82.5-

85.2; $[\alpha]_{\mathrm{D}}^{20^{\circ} \mathrm{C}}\left(\mathrm{CH}_{2} \mathrm{Cl}_{2}, \mathrm{c}=1.05 \mathrm{mg} / \mathrm{ml}\right)=+53.5^{\circ} . \mathrm{IR}(\mathrm{KBr}) \vee\left[\mathrm{cm}^{-1}\right] 781,838,1149,1719,1759,2875,2933$, 3198. ${ }^{1} \mathrm{H}$ NMR $\left(\mathrm{CDCl}_{3}\right) \delta[\mathrm{ppm}] 0.03\left(\mathrm{~s}, 3 \mathrm{H}, \mathrm{CH}_{3} \mathrm{Si}\right), 0.04\left(\mathrm{~s}, 3 \mathrm{H}, \mathrm{CH}_{3} \mathrm{Si}\right), 0.84(\mathrm{~s}, 9 \mathrm{H}, t-\mathrm{BuSi}), 0.87(\mathrm{t}, 3 \mathrm{H}, \mathrm{J}=7.2$ $\left.\mathrm{Hz}, \underline{\mathrm{CH}}_{3}\left(\mathrm{CH}_{2}\right)_{3}-\mathrm{O}\right), 1.19$ (d, 3H, J=6.3 Hz, $\left.\underline{\mathrm{CH}}_{3}-\mathrm{CH}(-\mathrm{OTBS})-\mathrm{CH}\right), 1.21-1.70$ (m, 7H), 1.90-2.3 (m, 3H), 2.71 (dd, $\left.1 \mathrm{H}, J_{1}=1.5, J_{2}=5.2 \mathrm{~Hz}, \mathrm{H}-3\right), 2.85\left(\mathrm{ddd}, 1 \mathrm{H}, J_{1}=4.2, J_{2}=8.8, J_{3}=14.1 \mathrm{~Hz}, \mathrm{H}-{ }^{`} 1\right), 3.10-3.40\left(\mathrm{~m}, 3 \mathrm{H}, \mathrm{O}-\underline{\mathrm{CH}}_{\underline{2}^{-}}\left(\mathrm{CH}_{2}\right)_{2}{ }^{-}\right.$ $\mathrm{CH}_{3}$ and H-3'), $3.62\left(\mathrm{dd}, 1 \mathrm{H}, J_{1}=2.0, J_{2}=9.6 \mathrm{~Hz}\right), 4.13(\mathrm{dq}, 1 \mathrm{H}, J=5.7 \mathrm{~Hz}), 6.13(\mathrm{~s}, 1 \mathrm{H}, \mathrm{NH}) . \mathrm{HRMS}\left([\mathrm{M}+\mathrm{H}]^{+}\right)$: Anal. calcd. for $\mathrm{C}_{21} \mathrm{H}_{40} \mathrm{NO}_{4} \mathrm{Si}$ : 398.2727; found: 398.2715.

\section{General Procedure B. Synthesis of 3-(S)-[1-(R)-(tert-Butyldimethylsilyloxy)-ethyl]-4-(R)-((1R/S)-3-(S/R)-} alkoxy-2-oxo-cyclohexyl)-allyloxalyl-azetidin-2-on (7a-h)

To an ice-cooled $\left(0^{\circ} \mathrm{C}\right)$ solution of 3-(S)-[1-(R)-(tert-Butyldimethylsilyloxy)-ethyl]-4- $(R)-((1 \mathrm{~S} / R)-3-$ $(R / S)$-alkoxy-2-oxo-cyclohexyl)-azetidin-2-one (4a-h) (44.2 mmol, $1 \mathrm{EQ})$ in dry THF (310 mL) AOK (allyl oxallylchloride, $17.5 \mathrm{~mL}, 3.3 \mathrm{EQ})$ was added. Next, $\mathrm{Et}_{3} \mathrm{~N}$ (27.0 mL, $4 \mathrm{EQ}$ ) in THF (27 mL) was added stepwise over 30 min at -5 to $0^{\circ} \mathrm{C}$ and the reaction mixture stirred first for 30 min at $0^{\circ} \mathrm{C}$ and then for $1.5 \mathrm{~h}$ at room temperature. Ethyl acetate $(800 \mathrm{~mL})$ was added to the reaction mixture and the precipitated solid was filtered off. The remaining solution was washed with $0.5 \mathrm{M} \mathrm{HCl}$ solution (600 $\mathrm{mL})$, a saturated solution of $\mathrm{NaHCO}_{3}(600 \mathrm{~mL})$ and a saturated solution of $\mathrm{NaCl}(600 \mathrm{~mL})$. The organic phase was dried over $\mathrm{MgSO}_{4}$ and the solvent evaporated under reduced pressure. The obtained oily product was purified by column chromatography.

3-(S)-[1-(R)-(tert-Butyldimethylsilyloxy)-ethyl]-4-(R)-((1S)-3-(R)-butoxy-2-oxo-cyclohexyl)-allyloxalyl-azetidin-2on $(7 e)$ 
Compound 7e was prepared from 4e according to General Procedure B. The obtained oily product was purified by

column chromatography (silica gel; ethyl acetate/hexane $=1 / 2$ ); yield: $79.7 \%$, yellow oil; $[\alpha]_{\mathrm{D}}^{20^{\circ} \mathrm{C}}\left(\mathrm{CH}_{2} \mathrm{Cl}_{2}, \mathrm{c}=\right.$ $1.02 \mathrm{mg} / \mathrm{ml})=-84.2^{\circ} . \mathrm{IR}(\mathrm{NaCl}) \vee\left[\mathrm{cm}^{-1}\right] 838,1213,1702,1806,2860,2934,2956,3383 . \mathrm{NMR}\left(\mathrm{CDCl}_{3}\right) \delta[\mathrm{ppm}]$ $-0.01\left(3 \mathrm{H}, \mathrm{s}, \mathrm{CH}_{3} \mathrm{Si}\right), 0.04\left(3 \mathrm{H}, \mathrm{s}, \mathrm{CH}_{3} \mathrm{Si}\right), 0.81(9 \mathrm{H}, \mathrm{s}, t-\mathrm{BuSi}), 0.87\left(\mathrm{t}, 3 \mathrm{H}, J=7.3 \mathrm{~Hz}, \underline{\mathrm{CH}}_{3}-\left(\mathrm{CH}_{2}\right)_{3}-\mathrm{O}\right), 1.29(\mathrm{~d}, 3 \mathrm{H}$, $\left.J=6 \mathrm{~Hz}, \underline{\mathrm{CH}}_{3}-\mathrm{CH}-\mathrm{OSi}\right), 1.14-1.79(\mathrm{~m}, 7 \mathrm{H}), 1.86-2.20(\mathrm{~m}, 3 \mathrm{H}), 3.11$ (deg.dd, 1H, J=3.1 Hz, H-3), 3.37 (t, $2 \mathrm{H}$, $\left.J=6.4 \mathrm{~Hz}, \mathrm{O}-\underline{\mathrm{CH}}_{2}-\left(\mathrm{CH}_{2}\right)_{2}-\mathrm{CH}_{3}\right), 3.48-3.62\left(\mathrm{~m}, 1 \mathrm{H}, \mathrm{H}^{\prime} 1\right), 3.69\left(\mathrm{dd}, 1 \mathrm{H}, J_{1}=3.0, J_{2}=5.0 \mathrm{~Hz}, \mathrm{H}-{ }^{\prime} 3\right), 4.32(\mathrm{dq}, 2 \mathrm{H}$, $\left.J_{1}=2.7, J_{2}=6.4 \mathrm{~Hz}, \mathrm{CH}_{3}-\underline{\mathrm{CH}}(-\mathrm{OTBS})-\mathrm{CH}-\right), 4.63\left(\mathrm{dd}, 2 \mathrm{H}, J_{1}=3.5, J_{2}=4.6 \mathrm{~Hz}, \mathrm{H}-4\right), 4.71-4.79$ (m, 2H, allyl), 5.22$5.42(\mathrm{~m}, 2 \mathrm{H} \text {, allyl), 5.82-6.00 (m, 1H, allyl). HRMS ([M+H] }]^{+}$): Anal. calcd. for $\mathrm{C}_{26} \mathrm{H}_{44} \mathrm{NO}_{7} \mathrm{Si}$ : 510.2887; found: 510.2881. HPLC purity: 91.01 area\%.

\section{3-(S)-[1-(R)-(tert-Butyldimethylsilyloxy)-ethyl]-4-(R)-((1R)-3-(S)-butoxy-2-oxo-cyclohexyl)-allyloxalyl-aze-} tidin-2-one (7f)

Compound $7 \mathbf{f}$ was prepared from $\mathbf{4 f}$ according to General Procedure $\mathbf{B}$. The obtained oily product was purified by column chromatography (silica gel; ethyl acetate/hexane $=1 / 2$ ); yield: $82.6 \%$, yellow oil; $[\alpha]_{\mathrm{D}}{ }^{20} \mathrm{C}\left(\mathrm{CH}_{2} \mathrm{Cl}_{2}, 1.02\right.$ $\mathrm{mg} / \mathrm{ml})=-22.0^{\circ} . \mathrm{IR}(\mathrm{NaCl}) \vee\left[\mathrm{cm}^{-1}\right] 838,1260,1391,1695,1757,1810,2859,2934,2956,3420 .{ }^{1} \mathrm{H}$ NMR $\left(\mathrm{CDCl}_{3}\right) \delta[\mathrm{ppm}]-0.06\left(\mathrm{~s}, 3 \mathrm{H}, \mathrm{CH}_{3} \mathrm{Si}\right),-0.02\left(\mathrm{~s}, 3 \mathrm{H}, \mathrm{CH}_{3} \mathrm{Si}\right), 0.75(\mathrm{~s}, 9 \mathrm{H}, t-\mathrm{BuSi}), 0.81\left(\mathrm{t}, 3 \mathrm{H}, J=7.2 \mathrm{~Hz}, \underline{\mathrm{CH}_{3}-}\right.$ $\left.\left(\mathrm{CH}_{2}\right)_{3}-\mathrm{O}\right), 1.11\left(\mathrm{~d}, 3 \mathrm{H}, \mathrm{J}=6.3 \mathrm{~Hz}, \underline{\mathrm{CH}}_{3}-\mathrm{CH}-\mathrm{OSi}\right), 1.26-1.69(\mathrm{~m}, 7 \mathrm{H}), 1.90-2.20(\mathrm{~m}, 3 \mathrm{H}), 3.06-3.28\left(\mathrm{~m}, 2 \mathrm{H}, \mathrm{O}^{-\mathrm{CH}_{2}} \underline{\underline{-}}^{-}\right.$ $\left.\left(\mathrm{CH}_{2}\right)_{2}-\mathrm{CH}_{3}\right), 3.29$ (deg. dd, 1H, J=3.4 Hz, H-`3), 3.51 (deg. dd, 1H, J=2.7 Hz, H-`3), 3.73 (ddd, 1H, $J_{1}=5.0$, $J_{2}=5.0, J_{2}=12.8 \mathrm{~Hz}, \mathrm{H}^{\prime} 1$ ) $)$ 4.16-4.28 (m, 2H, H-4 and $\left.\mathrm{CH}_{3}-\mathrm{CH}(-\mathrm{OTBS})-\mathrm{CH}-\right)$, 4.64-4.74 (m, 2H, allyl), 5.16-5.36 (m, 2H, allyl), 5.78-5.95 (m, 1H, allyl). HRMS $\left([\mathrm{M}+\mathrm{H}]^{+}\right)$: Anal. calcd. for $\mathrm{C}_{26} \mathrm{H}_{44} \mathrm{NO}_{7} \mathrm{Si}$ : 510.2887; found: 510.2898. 97.31 area\%.

\section{General Procedure C. Synthesis of allyl $(8 S / 8 R, 9 R)-10-(S)-[1-(R)-(t e r t-b u t y l d i m e t h y l s i l y l o x y)-e t h y l]-4-$ (S/R)-alkoxy-11-oxo-1-azatricyclo[7.2.0.0 $\left.{ }^{3,8}\right]$ undec-2-encarboxylate (8a-8h)}

Hydroquinone (1.46 g, 0.25 EQ) was added into a solution of 3-(S)-[1-(R)-(tert-butyldimethylsilyloxy)-ethyl]-4$(R)-((1 R / S)-3-(S / R)$-alkoxy-2-oxo-cyclohexyl)-allyloxalyl-azetidin-2-on (7a-7h) $(53.0 \mathrm{mmol}, 1 \mathrm{EQ})$ in xylene $(300$ 
$\mathrm{mL})$, and heated to boiling point. During refluxing, $\mathrm{P}(\mathrm{OEt})_{3}(46.0 \mathrm{~mL}, 265 \mathrm{mmol}, 5 \mathrm{EQ})$ was added in one portion and the reaction mixture stirred at reflux temperature for 2.5-3 h. After the reaction was complete the solvent was evaporated under reduced pressure to give an oily product, which was purified by column chromatography.

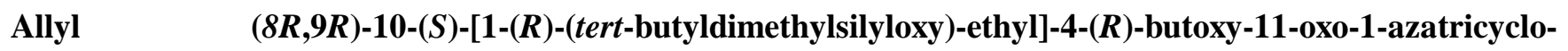
$\left[7.2 .0 .0^{3,8}\right]$ undec-2-encarboxylate $(8 \mathrm{e})$

Compound 8e was prepared from 7e according to General Procedure C. The obtained product was purified by column chromatography (silica gel; ethyl acetate/hexane $=1 / 6$ ); yield: $77.1 \%$, yellow oil; $[\alpha]_{\mathrm{D}}{ }^{20^{\circ} \mathrm{C}}\left(\mathrm{CH}_{2} \mathrm{Cl}_{2}, 0.95\right.$ $\mathrm{mg} / \mathrm{ml})=+35.8^{\circ} . \mathrm{IR}(\mathrm{NaCl}) \vee\left[\mathrm{cm}^{-1}\right] 777,835,1139,1320,1720,1784,2858,2933,3423 .{ }^{1} \mathrm{H} \mathrm{NMR}\left(\mathrm{CDCl}_{3}\right) \delta$ [ppm] 0.06 (s, 6H, $\left.\mathrm{CH}_{3} \mathrm{Si}\right), 0.87$ (s, 9H, t-BuSi), 0.87 (t, 3H, J=7.3 Hz, $\left.\underline{\mathrm{CH}}_{3}-\left(\mathrm{CH}_{2}\right)_{3}-\mathrm{O}\right), 1.23$ (d, 3H, J=6.1 Hz, $\left.\underline{\mathrm{CH}}_{3}-\mathrm{CH}_{2}-\mathrm{O}\right), 1.26-1.58(\mathrm{~m}, 7 \mathrm{H}), 1.70-1.90(\mathrm{~m}, 1 \mathrm{H}), 1.96-2.18(\mathrm{~m}, 2 \mathrm{H}), 3.06-3.20(\mathrm{~m}, 2 \mathrm{H}, \mathrm{H}-8$ and H-10), 3.26$3.44\left(\mathrm{~m}, 2 \mathrm{H}, \mathrm{O}-\underline{\mathrm{CH}}_{2}-\left(\mathrm{CH}_{2}\right)_{2}-\mathrm{CH}_{3}\right), 3.66\left(\mathrm{dd}, 1 \mathrm{H}, J_{1} \mathrm{~J}=2.9, J_{2}=7.2 \mathrm{~Hz}, \mathrm{H}-8\right), 4.15$ (deg. dq, 1H, J=6.3 Hz, $\mathrm{CH}_{3}-\underline{\mathrm{CH}}-$ OSi), 4.64-4.80 (m, 2H, allyl), 4.92 (deg. dd, 1H, J=2.8 Hz, H-4), 5.37 (m, 2H, allyl), 5.95 (m, 1H, allyl). HRMS $\left([\mathrm{M}+\mathrm{H}]^{+}\right)$: Anal. calcd. for $\mathrm{C}_{26} \mathrm{H}_{44} \mathrm{NO}_{5} \mathrm{Si}: 478.2989$; found: 478.2967. HPLC purity: 97.23 area\%.

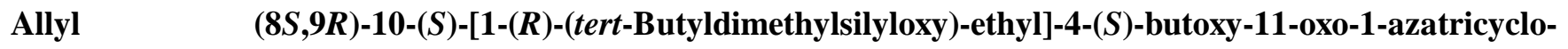
$\left[7.2 .0 .0^{3,8}\right]$ undec-2-encarboxylate $(8 f)$

Compound 8f was prepared from $7 \mathbf{f}$ according to General Procedure C. The obtained product was purified by column chromatography (silica gel; ethyl acetate/hexane $=1 / 6$ ); yield: $57.4 \%$, yellow oil; $[\alpha]_{\mathrm{D}}{ }^{20^{\circ} \mathrm{C}}\left(\mathrm{CH}_{2} \mathrm{Cl}_{2}, 1.06\right.$ $\mathrm{mg} / \mathrm{ml})=+7.5^{\circ} . \mathrm{IR}(\mathrm{NaCl}) \vee\left[\mathrm{cm}^{-1}\right] 778,836,1729,1781,2858,2931,2956,3433 .{ }^{1} \mathrm{H} \mathrm{NMR}\left(\mathrm{CDCl}_{3}\right) \delta[\mathrm{ppm}]$ 0.07 (s, 3H, $\left.\mathrm{CH}_{3} \mathrm{Si}\right), 0.08$ (s, 3H, $\left.\mathrm{CH}_{3} \mathrm{Si}\right), 0.87$ (s, 9H, t- BuSi), $0.91\left(\mathrm{t}, 3 \mathrm{H}, J=7.3 \mathrm{~Hz}, \mathrm{CH}_{3}-\left(\mathrm{CH}_{2}\right)_{3}-\mathrm{O}\right), 1.23(\mathrm{~d}, 3 \mathrm{H}$, $\left.J=6.1 \mathrm{~Hz}, \underline{\mathrm{CH}_{3}}-\mathrm{CH}_{2}-\mathrm{O}\right), 1.26-1.76(\mathrm{~m}, 7 \mathrm{H}), 1.78-1.12(\mathrm{~m}, 3 \mathrm{H}), 3.08-3.24(\mathrm{~m}, 2 \mathrm{H}, \mathrm{H}-8$ and H-10), $3.34(\mathrm{t}, 2 \mathrm{H}$, $\left.\mathrm{J}=6.5 \mathrm{~Hz}, \mathrm{O}-\underline{\mathrm{CH}}_{2}-\left(\mathrm{CH}_{2}\right)_{2}-\mathrm{CH}_{3}\right), 3.66\left(\mathrm{dd}, 1 \mathrm{H}, J_{1} \mathrm{~J}=3.1, J_{2}=10.3 \mathrm{~Hz}, \mathrm{H}-9\right), 4.21\left(\mathrm{deg} . \mathrm{dq}, 1 \mathrm{H}, \mathrm{J}=6.2 \mathrm{~Hz}, \mathrm{CH}_{3}-\underline{\mathrm{CH}}-\right.$ OSi), 4.62-4.84 (m, 2H, allyl), 5.04 (deg. dd, 1H, J=2.9 Hz, H-4), 5.21-5.49 (m, 2H, allyl), 5.95 (m, 1H, allyl). HRMS ([M+H] $\left.]^{+}\right)$: Anal. calcd. for $\mathrm{C}_{26} \mathrm{H}_{44} \mathrm{NO}_{5} \mathrm{Si}: 478.2989$; found: 478.2965. HPLC purity: 98.21 area\%

4. General Procedure D. Synthesis of allyl $(8 S / 8 R, 9 R)-10-(S)-[1-(R)-h y d r o x y e t h y l]-4-(S / R)$-alkoxy-11-oxo-1azatricyclo[7.2.0.0 $\left.{ }^{3,8}\right]$ undec-2-en-carboxylate $(9 \mathrm{a}-9 \mathrm{~h})$

A solution of TBAF (1 M solution in THF; $72.0 \mathrm{~mL}, 71.3 \mathrm{mmol}, 3 \mathrm{EQ})$ and AcOH (5.4 mL, $95.1 \mathrm{mmol}, 4 \mathrm{EQ})$ in

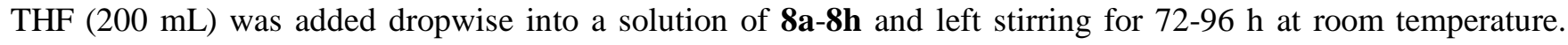


Ethylacetate $(700 \mathrm{~mL})$ was added and the reaction mixture washed with a saturated solution of $\mathrm{NaHCO}_{3}(500 \mathrm{~mL})$ and a saturated solution of $\mathrm{NH}_{4} \mathrm{Cl}(500 \mathrm{~mL})$, dried with $\mathrm{Na}_{2} \mathrm{SO}_{4}$ and evaporated under reduced pressure. The obtained oily product was purified by column chromatography.

Allyl $\quad(8 R, 9 R)-10-(S)-[1-(R)-h y d r o x y e t h y l]-4-(R)$-butoxy-11-oxo-1-azatricyclo[7.2.0.0 3,8$]$ undec-2-en-carboxylate (9e)

Compound 9e was prepared from 8e according to General Procedure D. The obtained product was purified by column chromatography (silica gel; ethyl acetate/hexane $=1 / 2$ ); yield: $70.6 \%$, yellow oil; $[\alpha]_{\mathrm{D}}{ }^{20^{\circ} \mathrm{C}}\left(\mathrm{CH}_{2} \mathrm{Cl}_{2}, 1.02\right.$ $\mathrm{mg} / \mathrm{ml})=0^{\circ} . \mathrm{IR}(\mathrm{NaCl}) \vee\left[\mathrm{cm}^{-1}\right] 1085,1181,1320,1720,1781,2870,2934,3440 .{ }^{1} \mathrm{H} \mathrm{NMR}\left(\mathrm{CDCl}_{3}\right) \delta[\mathrm{ppm}]$ 0.87 (t, 3H, J=7.3 Hz, $\left.\underline{\mathrm{CH}}_{3}-\left(\mathrm{CH}_{2}\right)_{3}-\mathrm{O}\right), 1.30$ (d, 3H, J=6.4 Hz, $\left.\underline{\mathrm{CH}}_{3}-\mathrm{CH}_{2}-\mathrm{O}\right), 1.26-1.60(\mathrm{~m}, 7 \mathrm{H}), 1.70-1.88$ (m, $\left.1 \mathrm{H}\right)$, 1.97-2.22 (m, 2H), 3.08-3.22 (m, 2H, H-8 and H-10), 3.26-3.45 (m, 2H, O- $\left.\mathrm{CH}_{2}-\left(\mathrm{CH}_{2}\right)_{2}-\mathrm{CH}_{3}\right), 3.72(\mathrm{dd}, 1 \mathrm{H}$, $\left.J_{1}=2.9, J_{2}=7.1 \mathrm{~Hz}, \mathrm{H}-8\right), 4.19$ (deg. dq, 1H, J=6.4 Hz, $\left.\mathrm{CH}_{3}-\underline{\mathrm{CH}}(-\mathrm{OH})-\mathrm{CH}\right), 4.63-4.82$ (m, 2H, allyl), 4.92 (deg. dd, 1H, J=2.8 Hz, H-4), 5.20-5.46 (m, 2H, allyl), 5.86-6.40 (m, 1H, allyl). HRMS $\left([\mathrm{M}+\mathrm{H}]^{+}\right)$: Anal. calcd. for $\mathrm{C}_{20} \mathrm{H}_{30} \mathrm{NO}_{5}$ : 364.2124; found: 364.2116. HPLC purity: 91.01 area\%.

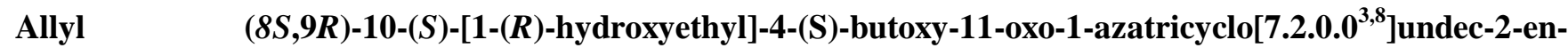
carboxylate (9f)

Compound 9f was prepared from $8 \mathbf{f}$ according to General Procedure D. The obtained product was purified by column chromatography (silica gel; ethyl acetate/hexane $=1 / 2$ ); yield: $68.7 \%$, yellow oil; $[\alpha]_{\mathrm{D}}{ }^{20^{\circ} \mathrm{C}}\left(\mathrm{CH}_{2} \mathrm{Cl}_{2}, 0.98\right.$ $\mathrm{mg} / \mathrm{ml})=-309^{\circ} . \mathrm{IR}(\mathrm{NaCl}) \vee\left[\mathrm{cm}^{-1}\right] 1086,1284,1719,1778,2871,2935,3451 .{ }^{1} \mathrm{H} \mathrm{NMR}\left(\mathrm{CDCl}_{3}\right) \delta[\mathrm{ppm}] 0.87(\mathrm{t}$, $\left.3 \mathrm{H}, J=7.3 \mathrm{~Hz}, \underline{\mathrm{CH}}_{3}-\left(\mathrm{CH}_{2}\right)_{3}-\mathrm{O}\right), 1.27\left(\mathrm{~d}, 3 \mathrm{H}, J=6.4 \mathrm{~Hz}, \underline{\mathrm{CH}}_{3}-\mathrm{CH}_{2}-\mathrm{O}\right), 1.26-1.66(\mathrm{~m}, 7 \mathrm{H}), 1.74-1.94(\mathrm{~m}, 1 \mathrm{H}), 1.98-$ $2.08(\mathrm{~m}, 2 \mathrm{H}), 3.14-3.38\left(\mathrm{~m}, 4 \mathrm{H}, \mathrm{O}-\underline{\mathrm{CH}}_{2}-\left(\mathrm{CH}_{2}\right)_{2}-\mathrm{CH}_{3}, \mathrm{H}-8\right.$ and $\left.\mathrm{H}-10\right), 4.10-4.26\left(\mathrm{~m}, 2 \mathrm{H}, \mathrm{H}-9\right.$ and $\mathrm{CH}_{3}-\underline{\mathrm{CH}}(-\mathrm{OH})-$ $\mathrm{CH}), 4.59-4.84(\mathrm{~m}, 2 \mathrm{H}$, allyl), 5.00 (deg. dd, 1H, J=2.9 Hz, H-4), 5.20-5.44 (m, 2H, allyl), 5.85-6.05 (m, 1H, allyl). HRMS ([M+H] $\left.]^{+}\right)$: Anal. calcd. for $\mathrm{C}_{20} \mathrm{H}_{30} \mathrm{NO}_{5}$ : 364.2124; found: 364.2126. HPLC purity: 94.65 area\%

5. General Procedure E. Synthesis of allyl $(8 S / 8 R, 9 R)-10-(E)$-ethylidene-4- $(S / R)$-alkoxy-11-oxo-1-azatricyclo[7.2.0.0 $\left.{ }^{3,8}\right]$ undec-2-en-carboxylate (10a-10h)

$\mathrm{PPh}_{3}(5.48 \mathrm{~g}, 20.9 \mathrm{mmol})$ and DEAD $(3.83 \mathrm{~mL}, 24.6 \mathrm{mmol})$ were added into the solution of 9a-9h $(20.9 \mathrm{mmol})$ in $\mathrm{CH}_{2} \mathrm{Cl}_{2}(180 \mathrm{~mL})$ at $0^{\circ} \mathrm{C}$ during stirring. The temperature was left to rise to room temperature and the reaction 
mixture evaporated under reduced pressure. The triphenylphosphine oxide was precipitated from the reaction mixture using diethyl ether and compound purified by column chromatography.

Allyl $(8 S, 9 R)-10-(E)$-ethylidene-4-(S)-methoxy-11-oxo-1-azatricyclo[7.2.0.0 ${ }^{3,8}$ undec-2-en-carboxylate (10a) Compound 10a was prepared from 9a according to General Procedure E. The obtained product was purified by column chromatography ( silica gel; diethyleter/hexane $=1 / 2$ ); yield: $70.0 \%$, white crystals; m.p. $76-79^{\circ} \mathrm{C}$; $[\alpha]_{\mathrm{Na}}^{20^{\circ} \mathrm{C}}\left(\mathrm{CH}_{2} \mathrm{Cl}_{2}, 10.0 \mathrm{mg} / \mathrm{ml}\right)=+70.8^{\circ} . \mathrm{IR}(\mathrm{KBr}) \vee\left[\mathrm{cm}^{-1}\right] 3246,2945,2828,1760,1713,1629,1533,1418$, 1339, 1314, 1212, 997, 958, 820. ${ }^{1} \mathrm{H}$ NMR $\left(\mathrm{DMSO}-d_{6}\right) \delta[\mathrm{ppm}] 1.24-1.63(\mathrm{~m}, 6 \mathrm{H}), 1.83\left(\mathrm{~d}, 3 \mathrm{H}, J=6.3 \mathrm{~Hz}, \underline{\mathrm{CH}}_{3}{ }^{-}\right.$ $\mathrm{CH}=), 3.25\left(\mathrm{~s}, 3 \mathrm{H},-\mathrm{OCH}_{3}\right), 3.25-3.28(\mathrm{~m}, 1 \mathrm{H}), 4.67-4.83(\mathrm{~m}, 3 \mathrm{H}, \mathrm{H}-9), 4.97$ (t, 1H, J=2.9 Hz, H-4), 5.28 (dq, 1H, $\left.J=10.4 \mathrm{~Hz}, 1.4 \mathrm{~Hz}, \mathrm{CH}_{3}-\underline{\mathrm{CH}}=\right), 5.45(\mathrm{dd}, 1 \mathrm{H}, J=17.3,1.4 \mathrm{~Hz}), 5.93-6.06(\mathrm{~m}, 1 \mathrm{H}), 6.51\left(\mathrm{dq}, 1 \mathrm{H}, J_{1}=7.1, J_{2}=1.7\right.$ Hz). ${ }^{13} \mathrm{C}$ NMR $\left(\mathrm{DMSO}-d_{6}\right) \delta[\mathrm{ppm}] 14.4,20.0,30.8,32.4,44.9,56.1,60.9,62.2,72.4,118.7,127.2,129.5$, 131.5, 140.1, 147.9, 161.2, 171.4. HRMS $\left([\mathrm{M}+\mathrm{H}]^{+}\right)$: Anal. calcd. for $\mathrm{C}_{17} \mathrm{H}_{22} \mathrm{NO}_{4}$ : 304.1549; found: 304.1548 . HPLC purity: 99.15 area\%.

\section{Allyl $(8 R, 9 R)$-10- $(E)$-ethylidene-4- $(R)$-ethoxy-11-oxo-1-azatricyclo[7.2.0.0 $\left.{ }^{3,8}\right]$ undec-2-en-carboxylate (10b)}

Compound 10b was prepared from $\mathbf{9 b}$ according to General Procedure E. The obtained product was purified by column chromatography (silica gel; ethyl acetate/hexane $=1 / 2$ ); yield: $55.0 \%$, yellow oil; $[\alpha]_{\mathrm{Na}}{ }^{20^{\circ} \mathrm{C}}\left(\mathrm{CH}_{2} \mathrm{Cl}_{2}, 1.085\right.$ $\mathrm{mg} / \mathrm{ml})=-5.8^{\circ} . \mathrm{IR}(\mathrm{KBr}) \vee\left[\mathrm{cm}^{-1}\right] 2974,2935,2868,1776,1719,1632,1445,1368,1348,1319,1285,1201$, 1120, 977, 924. ${ }^{1} \mathrm{H}$ NMR $\left(\mathrm{CDCl}_{3}\right) \delta[\mathrm{ppm}] 1.13\left(\mathrm{t}, 3 \mathrm{H}, J=7 \mathrm{~Hz}, \underline{\mathrm{CH}_{3}}{ }^{-} \mathrm{CH}_{2}-\mathrm{O}\right), 1.82\left(\mathrm{dd}, 3 \mathrm{H}, J=7 \mathrm{~Hz}, 1 \mathrm{~Hz}, \underline{\mathrm{CH}_{3}-}\right.$ $\mathrm{CH}=), 1.4-2.2(\mathrm{~m}, 6 \mathrm{H}), 3.20\left(\mathrm{~m}, 1 \mathrm{H}, J_{1}=4 \mathrm{~Hz}, J_{2}=7 \mathrm{~Hz}, \mathrm{H}-8\right), 3.44\left(\mathrm{~m}, 2 \mathrm{H}, J=7 \mathrm{~Hz}, \mathrm{CH}_{3}-\underline{\mathrm{CH}}_{2}-\mathrm{O}\right), 4.29(\mathrm{~d}, 1 \mathrm{H}, J=$ $7 \mathrm{~Hz}, \mathrm{H}-9), 4.77$ (m, 2H, allyl), 4.99 (t, 1H, J=3 Hz, H-4), 5.36 (m, 2H, allyl), 5.98 (m, 1H, allyl), 6.40 (dq, 1H, $\left.J=7 \mathrm{~Hz}, \mathrm{CH}_{3}-\underline{\mathrm{CH}}=\right)$. HRMS $\left([\mathrm{M}+\mathrm{H}]^{+}\right)$: Anal. calcd. for $\mathrm{C}_{18} \mathrm{H}_{24} \mathrm{NO}_{4}: 318.1705$; found: 318.1700. HPLC purity: 96.87 area\%.

\section{Allyl (8S,9R)-10-(E)-ethylidene-4-(S)-ethoxy-11-oxo-1-azatricyclo[7.2.0.0 $\left.{ }^{3,8}\right]$ undec-2-en-carboxylate (10c)}

Compound 10c was prepared from 9c according to General Procedure E. The obtained product was purified by column chromatography ( silica gel; ethyl acetate/hexane $=1 / 2$ ); yield: $80.9 \%$, yellow oil; $[\alpha]_{\mathrm{Na}}^{20^{\circ} \mathrm{C}}\left(\mathrm{CH}_{2} \mathrm{Cl}_{2}, 1.01\right.$ $\mathrm{mg} / \mathrm{ml})=+96.7 . \mathrm{IR}(\mathrm{KBr}) \vee\left[\mathrm{cm}^{-1}\right] 2973,2938,2866,1772,1718,1633,1445,1317,1281,1167,1090 .{ }^{1} \mathrm{H}$ NMR $\left(\mathrm{CDCl}_{3}\right) \delta[\mathrm{ppm}] 1.19\left(\mathrm{t}, 3 \mathrm{H}, J=7.0 \mathrm{~Hz}, \underline{\mathrm{CH}_{3}}-\mathrm{CH}_{2}-\mathrm{O}\right), 1.84\left(\mathrm{dd}, 3 \mathrm{H}, J=7.2 \mathrm{~Hz}, 0.9 \mathrm{~Hz}, \underline{\mathrm{CH}_{3}}-\mathrm{CH}=\right), 1.40-2.09(\mathrm{~m}$, 
$6 \mathrm{H}), 3.27-3.35(\mathrm{~m}, 1 \mathrm{H}, \mathrm{H}-8), 3.37-3.46\left(\mathrm{~m}, 2 \mathrm{H}, \mathrm{CH}_{3}-\underline{\mathrm{CH}}_{2}-\mathrm{O}\right), 4.79$ (d, 1H, J=9.8 Hz, H-9), 4.67-4.88 (m, 2H, allyl), 5.08 (t, 1H, J=2.9 Hz, H-4), 5.26-5.31 (m, 1H, allyl), 5.42-5.49 (m, 1H, allyl), 5.93-6.06 (m, 1H, allyl), 6.51 (dq, $1 \mathrm{H}, J_{1}=7.2 \mathrm{~Hz}, J_{2}=1.7 \mathrm{~Hz}, \mathrm{CH}_{3}-\underline{\mathrm{CH}}=$ ). HRMS $\left([\mathrm{M}+\mathrm{H}]^{+}\right)$: Anal. calcd. for $\mathrm{C}_{18} \mathrm{H}_{24} \mathrm{NO}_{4}$ : 318.1705 ; found: 318.1693. HPLC purity: 98.19 area\%.

Allyl $\quad(8 S, 9 R)-10-(E)$-ethylidene-4-(S)-isopropoxy-11-oxo-1-azatricyclo[7.2.0.0 $\left.{ }^{3,8}\right]$ undec-2-en-carboxylate (10d)

Compound 10d was prepared from 9d according to General Procedure E. The obtained product was purified by column chromatography (silica gel; ethyl acetate/hexane $=1 / 4$ ); yield: $74.0 \%$, yellow oil; $[\alpha]_{\mathrm{Na}}{ }^{20^{\circ} \mathrm{C}}\left(\mathrm{CH}_{2} \mathrm{Cl}_{2}, 1.13\right.$ $\mathrm{mg} / \mathrm{ml})=+86.4 . \mathrm{IR}(\mathrm{KBr}) \vee\left[\mathrm{cm}^{-1}\right] 2970,2938,1772,1717,1632,1445,1368,1316,1281,1164,1017 .{ }^{1} \mathrm{H}$ NMR $\left(\mathrm{CDCl}_{3}\right) \delta[\mathrm{ppm}] 1.13\left(\mathrm{~d}, 3 \mathrm{H}, J=6.1 \mathrm{~Hz}, \underline{\mathrm{CH}}_{3}-\mathrm{CH}-\mathrm{O}\right), 1.14\left(\mathrm{~d}, 3 \mathrm{H}, J=6.1 \mathrm{~Hz}, \underline{\mathrm{CH}}_{3}-\mathrm{CH}-\mathrm{O}\right), 1.83\left(\mathrm{dd}, 3 \mathrm{H}, J_{1}=7.2 \mathrm{~Hz}\right.$ $\left.J_{2}=0.9 \mathrm{~Hz}, \underline{\mathrm{CH}}_{3}-\mathrm{CH}=\right), 1.41-1.60(\mathrm{~m}, 2 \mathrm{H}), 1.81-2.00(\mathrm{~m}, 4 \mathrm{H}), 3.28-3.37(\mathrm{~m}, 1 \mathrm{H}, \mathrm{H}-8), 3.48-3.60(\mathrm{~m}, 1 \mathrm{H}, J=6.1 \mathrm{~Hz}$ , $\left.\mathrm{CH}_{3}-\underline{\mathrm{CH}}-\mathrm{O}\right), 4.67-4.88$ (m, 2H, allyl), 4.79 (d, 1H, J=11.3 Hz, H-9), 5.18 (t, 1H, J=2.9 Hz, H-4), 5.26-5.31 (m, $1 \mathrm{H}$, allyl), 5.42-5.49 (m, 1H, allyl), 5.93-6.06 (m, 1H, allyl), 6.51 (dq, $\left.1 \mathrm{H}, J_{1}=7.2 \mathrm{~Hz}, J_{2}=1.7 \mathrm{~Hz}, \mathrm{CH}_{3}-\underline{\mathrm{CH}}=\right)$. HRMS $\left([\mathrm{M}+\mathrm{H}]^{+}\right)$: Anal. calcd. for $\mathrm{C}_{19} \mathrm{H}_{26} \mathrm{NO}_{4}$ : 332.1862; found: 332.1851. HPLC purity: 98.26 area\% .

Allyl $(8 R, 9 R)$-10-(E)-ethylidene-4-(R)-butoxy-11-oxo-1-azatricyclo[7.2.0.0 $\left.{ }^{3,8}\right]$ undec-2-en-carboxylate (10e)

Compound 10e was prepared from 9e according to General Procedure E. The obtained product was purified by column chromatography (silica gel; ethyl acetate/hexane $=1 / 4$ ); yield: $60.6 \%$, yellow oil; $[\alpha]_{\mathrm{D}}{ }^{20^{\circ} \mathrm{C}}\left(\mathrm{CH}_{2} \mathrm{Cl}_{2}, 1.08\right.$ $\mathrm{mg} / \mathrm{ml})=-26.5^{\circ} . \mathrm{IR}(\mathrm{NaCl}) \vee\left[\mathrm{cm}^{-1}\right] 761,1733,2934,3374 .{ }^{1} \mathrm{H} \mathrm{NMR}\left(\mathrm{CDCl}_{3}\right) \delta[\mathrm{ppm}] 0.87(\mathrm{t}, 3 \mathrm{H}, J=7.3 \mathrm{~Hz}$ $\left.\underline{\mathrm{CH}}_{3}-\left(\mathrm{CH}_{2}\right)_{3}-\mathrm{O}\right), 1.21-1.64(\mathrm{~m}, 7 \mathrm{H}), 1.82\left(\mathrm{dd}, 3 \mathrm{H}, J_{1}=1.0, J_{2}=7.3 \mathrm{~Hz}, \mathrm{CH}_{3}-\mathrm{CH}=\right), 1.96-2.28(\mathrm{~m}, 1 \mathrm{H}), 3.19$ (deg. ddd, 1H, J=6.2 Hz, H-9), 3.26-3.48 (m, 2H, O- $\left.\underline{\mathrm{CH}}_{2}-\left(\mathrm{CH}_{2}\right)_{2}-\mathrm{CH}_{3}\right), 4.29$ (d, 1H, J=6.7 Hz, H-9), 4.68-4.86 (m, 2H, allyl), 4.95 (deg. dd, 1H, J=2.8 Hz, H-4), 5.24-5.52 (m, 2H, allyl), 5.90-6.07 (m, 1H, allyl), 6.40 (dq, 1H, $J_{1}=1.7$, $\left.J_{2}=7.2 \mathrm{~Hz}, \mathrm{CH}_{3}-\underline{\mathrm{CH}}=\right)$. HRMS $\left([\mathrm{M}+\mathrm{H}]^{+}\right)$: Anal. calcd. for $\mathrm{C}_{20} \mathrm{H}_{30} \mathrm{NO}_{6}: 346.2018$; found: 346.2026. HPLC purity: 90.37 area\%.

\section{Allyl $(8 S, 9 R)-10$ - $(E)$-ethylidene-4-(S)-butoxy-11-oxo-1-azatricyclo[7.2.0.0 $\left.{ }^{3,8}\right]$ undec-2-en-carboxylate (10f)}

Compound 10f was prepared from 9f according to General Procedure E. The obtained product was purified by column chromatography (silica gel; ethyl acetate/hexane $=1 / 4$ ); yield: $51.4 \%$, yellow oil; $[\alpha]_{\mathrm{D}}{ }^{20^{\circ} \mathrm{C}}\left(\mathrm{CH}_{2} \mathrm{Cl}_{2}, 1.065\right.$ 
$\mathrm{mg} / \mathrm{ml})=+107.9^{\circ} . \mathrm{IR}(\mathrm{NaCl}) \vee\left[\mathrm{cm}^{-1}\right] 1091,1721,1770,2871,2935,3412 .{ }^{1} \mathrm{H} \mathrm{NMR}\left(\mathrm{CDCl}_{3}\right) \delta[\mathrm{ppm}] 0.90(\mathrm{t}$, $\left.3 \mathrm{H}, J=7.3 \mathrm{~Hz}, \underline{\mathrm{CH}}_{3}-\left(\mathrm{CH}_{2}\right)_{3}-\mathrm{O}\right), 1.20-1.64(\mathrm{~m}, 9 \mathrm{H}), 1.82\left(\mathrm{dd}, 3 \mathrm{H}, J_{1}=0.9, J_{2}=7.2 \mathrm{~Hz}, \underline{\mathrm{CH}}_{3}-\mathrm{CH}=\right), 1.74-1.92(\mathrm{~m}, 1 \mathrm{H})$, 1.96-2.27 (m, 1H), 3.11-3.40 (m, 2H, O- $\left.\mathrm{CH}_{2}-\left(\mathrm{CH}_{2}\right)_{2}-\mathrm{CH}_{3}\right), 4.62-4.88$ (m, 3H, allyl and H-9), 5.02 (deg. dd, 1H, $J=2.8 \mathrm{~Hz}, \mathrm{H}-4), 5.26\left(\mathrm{~m}, 1 \mathrm{H}\right.$, allyl), $5.43\left(\mathrm{~m}, 1 \mathrm{H}\right.$, allyl), 5.87-6.06 (m, 1H, allyl), 6.49 (dq, $1 \mathrm{H}, J_{1}=1.7, J_{2}=7.2$ $\left.\mathrm{CH}_{3}-\underline{\mathrm{CH}}=\right)$. HRMS ([M+H] $\left.]^{+}\right)$: Anal. calcd. for $\mathrm{C}_{20} \mathrm{H}_{28} \mathrm{NO}_{4}$ : 346.2018; found: 346.2006. 96.99 area\%.

Allyl $\quad(8 S, 9 R)-10-(E)$-ethylidene-4-(S)-(2'-fluoroethoxy)-11-oxo-1-azatricyclo[7.2.0.0 $\left.{ }^{3,8}\right]$ undec-2-ene-2carboxylate (10g)

Compound 10g was prepared from 9g according to General Procedure E. The obtained product was purified with column chromatography (silicagel; ethyl acetate/hexane $=1 / 1$ ); yield: $75 \% .{ }^{1} \mathrm{H}$ NMR $\left(\mathrm{CDCl}_{3}\right) \delta[\mathrm{ppm}] 1.31-2.12$ $(\mathrm{m}, 6 \mathrm{H}), 1.84\left(\mathrm{dd}, 3 \mathrm{H}, \mathrm{J}_{1}=0.9, \mathrm{~J}_{2}=7.2 \mathrm{~Hz}\right), 3.34(\mathrm{~m}, 1 \mathrm{H}), 3.57(\mathrm{~m}, 1 \mathrm{H}), 3.67(\mathrm{~m}, 1 \mathrm{H}), 4.46(\mathrm{~m}, 1 \mathrm{H}), 4.62(\mathrm{~m}$ $1 \mathrm{H}), 4.67-4.87(\mathrm{~m}, 3 \mathrm{H}), 5.13(\mathrm{t}, 1 \mathrm{H}, J=2.9 \mathrm{~Hz}), 5.29(\mathrm{~m}, 1 \mathrm{H}), 5.46(\mathrm{~m}, 1 \mathrm{H}), 6.00(\mathrm{~m}, 1 \mathrm{H}), 6.52\left(\mathrm{dd}, 1 \mathrm{H}, J_{1}=1.7\right.$ $\left.J_{2}=7.2 \mathrm{~Hz}\right) . . \mathrm{MS}(\mathrm{FAB}, \mathrm{m} / \mathrm{z}) 336\left(\mathrm{MH}^{+}\right)$.

\footnotetext{
Allyl $\quad(8 S, 9 R)-10-(E)$-ethylidene-4-(S)-(2'-cianoethoxy)-11-oxo-1-azatricyclo[7.2.0.0 $\left.{ }^{3,8}\right]$ undec-2-ene-2carboxylate (10h) Compound 10h was prepared from 9h according to General Procedure E. The obtained product was purified with column chromatography (silicagel; ethyl acetate/cyclohexane = 1/1); yield: $66 \%$. ${ }^{1} \mathrm{H}$ NMR $\left(\mathrm{CDCl}_{3}\right) \delta[\mathrm{ppm}]$ 1.20-1.89 (m, 5H), $1.83(\mathrm{dd}, 1.0, J=7.2 \mathrm{~Hz}, 3 \mathrm{H}), 2.05(\mathrm{~m}, 1 \mathrm{H}), 2.60(\mathrm{~m}, 2 \mathrm{H}), 3.37(\mathrm{~m}$, 1H), $3.58(\mathrm{~m}, 2 \mathrm{H}), 4.71(\mathrm{~m}, 1 \mathrm{H}), 4.82(\mathrm{~m}, 2 \mathrm{H}), 5.12(\mathrm{t}, J=2.9 \mathrm{~Hz}, 1 \mathrm{H}), 5.28(\mathrm{~m}, 1 \mathrm{H}), 5.45(\mathrm{~m}, 1 \mathrm{H}), 5.99(\mathrm{~m}, 1 \mathrm{H})$, $6.51\left(\mathrm{dq}, J_{1}=1.7, J_{2}=7.2 \mathrm{~Hz}, 1 \mathrm{H}\right) .{ }^{13} \mathrm{C} \mathrm{NMR}\left(\mathrm{CDCl}_{3}\right) \delta[\mathrm{ppm}] 15.2,19.1,19.9,30.7,32.6,44.9,61.0,62.8,65.8$, 71.2, 118.0, 118.8, 127.6, 129.9, 131.3, 140.0, 147.0, 161.1, 171.3. MS (FAB, m/z) $343\left(\mathrm{MH}^{+}\right)$.
} 